\title{
Is State Anhedonia Characteristic of Parkinson's Disease?
}

\author{
Gwenolé Loas $^{1 *}$, Pierre Krystkowiak ${ }^{2,3}$ \\ ${ }^{1}$ Department of Psychiatry \& Laboratory of Psychiatric Research (ULB 266), Cliniques Universitaires de \\ Bruxelles, Hôpital Erasme, Université Libre de Bruxelles (ULB), Bruxelles, Belgium \\ ${ }^{2}$ Department of Neurology, Amiens University Hospital, Amiens, France \\ ${ }^{3}$ Laboratoire de Neurosciences Fonctionnelles et Pathologie (LNFP), University of Picardie Jules Verne (UPJV), \\ Amiens, France. \\ Email: "gwenole.loas@erasme.ulb.ac.be
}

Received 26 October 2015; accepted 14 November 2015; published 19 November 2015

Copyright @ 2015 by authors and Scientific Research Publishing Inc.

This work is licensed under the Creative Commons Attribution International License (CC BY).

http://creativecommons.org/licenses/by/4.0/

(c) (1) Open Access

\section{Abstract}

Anhedonia, the lowered ability to experience pleasure, is one of the non-motor symptoms in Parkinson's disease. Recently, the distinction between consummatory and anticipatory anhedonia has been proposed and anhedonia, notably in PD, could constitute a stable characteristic (anhedonia-trait) or secondary symptom (anhedonia-state). Several studies, using healthy control groups, reported high state consummatory and anticipatory anhedonia in PD using the Snaith Hamilton Pleasure Scale (SHAPS), but when control groups included subjects with different illnesses no significant differences were reported. The aim of the present study was to compare PD subjects with subjects presenting a non-Parkinson motor neurological disease on the anhedonia subscale of the Beck Depression Inventory (BDI-II). This subscale rated consummatory and anticipatory anhedonia state. No significant difference was reported. This result confirmed that PD subjects were not characterized by high levels of state anhedonia when the subjects were compared to subjects with a different disease. Contrary to trait consummatory anhedonia, state anhedonia could be nonspecific to Parkinson's disease.

\section{Keywords}

Parkinson's Disease, Anhedonia, Consummatory, Anticipatory, Depression, BDI-II

\footnotetext{
${ }^{*}$ Corresponding author.
} 


\section{Introduction}

Parkinson's disease (PD) is a motor system disorder, which is the result of the loss of dopamine-producing brain cells. PD is characterized by four primary symptoms: tremor, rigidity, bradykinesia and postural instability. Among the other symptoms depression and emotional deficits as apathy or anhedonia have high prevalence. The UK Parkinson's Disease Society Brain Bank (UKPDSBB) clinical diagnostic criteria are routinely used for the diagnosis. Several risk factors are identified: age (around age 60 or older), heredity, sex (men), exposure to toxins (herbicides, pesticides).

As underlined by Dyduch and Zaluska [1] some symptoms of depression and notably anhedonia, in Parkinson's disease (PD), present firstly a common neurobiological pathway and secondly a clinically overlap. Anhedonia, the lowered ability to experience pleasure, is a frequent symptom in Parkinson's disease (PD) with a prevalence ranging from $4.7 \%$ to $45.7 \%$ using the cut off of 3 on the Snaith-Hamilton Pleasure Scale (SHAPS) [2]. Compared with controls, higher scores on anhedonia rating scales were reported in PD [3]. One of the main problems in the exploration of the role of anhedonia in PD is its specificity. High anhedonia levels can be explained firstly by socio-demographical variables (e.g. age, gender, educational level) and secondly by clinical variables such as depression or hospitalisation [2]. Thus, to explore the specificity on anhedonia in PD it is necessary to control for the potential effects of the socio-demographical variables as well as the depressive level or the status of patients (in or outpatients). Moreover, the choice of the control group is very important. The specificity of anhedonia in PD can be established only with the use of controls presenting a non-Parkinson motor neurological disease. If the control group included only healthy subjects then any potential significant differences found between the healthy group and PD group would be explained only by the fact that the patients were ill, independently of the type of disease. If the control group included only patients with motor symptoms secondary to a non-Parkinson neurological disease then any potential significant difference would be explained by the Parkinson disease.

Of the seven studies [4]-[10] that have compared anhedonia rating scales scores between PD and controls, only one study used a control group of non-Parkinson motor neurological disease [10], the others having used healthy subjects group [5]-[9] or subjects with osteoarthritis [4].

Recently, two distinctive types of pleasure deficits have been proposed [11] [12]. Anticipatory anhedonia refers to the inability to anticipate pleasure for a future reward or "wanting", whereas consummatory anhedonia refers to the inability to experience pleasure related to online experience in response to a specific stimulus or "liking". Only three studies [9] [10] [13] have explored anticipatory and consummatory anhedonia in PD using the Temporal experience of pleasure scale (TEPS, [11]) or two subscales of the revised Physical Anhedonia Scale (PAS, [14]) and reported higher consummatory anhedonia in PD comparatively to healthy subjects or nonParkinson motor neurological disease.

Independently of anticipatory and consummatory types of anhedonia, anhedonia could constitute either a state or a trait. These two types of anhedonia are evaluated using different rating scales including different scoring instructions that rate either recent hedonic situations or long-term hedonic experiences. The SHAPS [15] or the anhedonia subscale of the Beck Depression Inventory-II [16] rates anhedonia state although the PAS, the Social Anhedonia Scale (SAS) [14] or the TEPS rates anhedonia trait. However, several studies in various groups of clinical or non-clinical subjects [11] [17]-[19] have examined the relationships between the TEPS and the SHAPS, PAS, SAS or BDI-II anhedonia subscale. The results found that the SHAPS and the BDI-II anhedonia subscale rate consummatory and anticipatory anhedonia state especially, the PAS rates consummatory and anticipatory anhedonia trait and the SAS rates anticipatory anhedonia trait. Thus, the use of the SHAPS, BDI-II anhedonia subscale, TEPS, PAS and SAS allows distinguishing state or trait of anhedonia and anticipatory or consummatory anhedonia.

Six studies [4] [6]-[10] have compared the scores on the SHAPS, which rates anhedonia state, or the prevalence of anhedonia using the cut off of 3 on this scale, between PD patients and controls. Four of these studies [6]-[9] reported significant differences and two [4] [10] non-significant differences. It is interesting to note that the two studies that reported non-significant differences used non-healthy control group (osteoarthritis or nonParkinson motor neurological disease).

Thus, the existence of state hedonic deficit remains an open question in PD. The aim of the present study was to explore state anhedonia, using the BDI-II anhedonia subscale, in PD compared to patients with a non-Parkinson motor neurological disease. 


\section{Methods}

\subsection{Participants}

For the present study we used two samples of a previously published study [10]. Thus we presented only the main characteristics of these two samples (see Table 1). The study included 49 outpatients (28 males, 21 females, mean age 64.84 years, SD $=10.84$ ) with idiopathic PD according to the UKPDSBB (United Kingdom Parkinson's Disease Brain Bank) criteria and 40 patients (18 males, 22 females, mean age 60.1 years old, SD = 12.07 ) with a non-PD motor neurological disease (OND). All subjects were outpatients and the study was performed at follow-up visits during regular treatment with antiparkinsonian drugs. There was no significant difference on age, gender or level of education between the two groups. The study was approved by our local Ethics Committee and in accordance with the Helsinki declaration, each subject signed an informed consent form before starting the study. The control group comprised 40 outpatients with the following motor neurological diseases: cervical dystonia $(N=17)$, hemifacial spasm $(N=8)$, blepharospasm $(N=7)$, writer's cramp $(N=4)$, cerebellar ataxia $(\mathrm{N}=2)$, myoclonus $(\mathrm{N}=1)$ and congenital torticollis $(\mathrm{N}=1)$. As dystonia was considered as a basal ganglia disorder contrary to the diagnoses of the other controls, the two groups were compared on the socio-demographic, clinical and psychometrical variables. No significant differences were found.

\subsection{Rating Scales}

The subjects of the two groups filled out several questionnaires including the BDI-II. The BDI-II is the 1996 revision of the classic BDI [20]. Each of the 21 items corresponding to a symptom of depression is summed to give a single score for the BDI-II. Total score ranges from 0 to 63 and higher total scores indicate more severe depressive symptoms. There is a four-point scale for each item ranging from 0 to 3 . Cut score guidelines for the BDI-II are given with the recommendation that thresholds be adjusted based on the characteristics of the sample, and the purpose for use of the BDI-II. Total score of 0-13 is considered minimal range, 14 - 19 is mild, 20 - 28 is moderate, and 29 - 63 is severe.The French version of the BDI-II has satisfactory psychometric properties [21]. The scoring instructions of the BDI-II explain that the subject must take into account the last two weeks for the responses. (For the SHAPS, the period of time is the last few days).

For the present study we use the anhedonia subscale of the BDI-II that contains three items (Item 4 "I don't get satisfaction out of anything anymore"; item 12 "I have lost all interest in other people and don't care about themat all" and item 21 "I have lost interest in sex completely"). This subscale has been validated by Joiner et al. [16]. The remaining $18 \mathrm{BDI}-\mathrm{II}$ items were also totalled to measure non-anhedonic depressive symptoms.

\subsection{Statistical Analyses}

The two groups were compared for the two rating scales using non-parametric Mann \& Whitney tests. Statistical tests were two-tailed with $\mathrm{p}<0.05$.

\section{Results}

The two groups did not differ significantly on the anhedonia subscale score of the BDI-II. The mean scores of the scale $(\mathrm{SD})$ of the PD and controls were $2.39(\mathrm{SD}=1.71)$ and $2.12(\mathrm{SD}=1.99)$, respectively $(\mathrm{U}=847, \mathrm{p}=$

Table 1. Comparison between Parkinson’s disease (PD) subjects and subjects with other neurological disease (OND).

\begin{tabular}{cccc}
\hline & PD $(\mathrm{n}=49)$ & OND $(\mathrm{n}=40)$ & $\mathrm{p}$ \\
\hline Gender & $57 \%$ males $(\mathrm{n}=28)$ & $45 \%$ males $(\mathrm{n}=18)$ & 0.25 \\
Age (years) & $64.84(\mathrm{sd}=10.84)$ & $60.1(\mathrm{SD}=12.07)$ & 0.06 \\
Level of education(I, II, III) & $22 / 14 / 13$ & $13 / 14 / 13$ & 0.67 \\
18-BDI-II & $16.12(\mathrm{SD}=7.73)$ & $12.87(\mathrm{SD}=9.94)$ & 0.03 \\
Anhedonia-BDI-II & $2.39(\mathrm{SD}=1.71)$ & $2.12(\mathrm{SD}=1.99)$ & 0.26 \\
Duration of disease (years) & $7.37(\mathrm{SD}=4.56)$ & & \\
Age at onset (years) & $56.7(\mathrm{SD}=10.44)$ & & \\
\hline
\end{tabular}

${ }^{\mathrm{a}}$ Level of education: I < baccalaureate, II = baccalaureate; III > baccalaureate; baccalaureate corresponds to the end of secondary school studies, i.e. 12 years of accredited education after the age of 6; BDI-II: Beck Depression Inventory-II. 
0.26). Concerning the 18-BDI, the PD had significantly higher scores than the controls. The mean scores of the scale $(\mathrm{SD})$ of the $\mathrm{PD}$ and controls were $16.12(\mathrm{SD}=7.73)$ and $12.87(\mathrm{SD}=9.94)$, respectively $(\mathrm{U}=724, \mathrm{p}=$ 0.034).

\section{Discussion}

PD subjects were not characterized by higher levels of anhedonia state, as rated by the anhedonia subscale of the BDI-II, compared with subjects presenting a non-Parkinson motor neurological disease. The two groups were not significantly different for age, gender, educational level. The control group included non-Parkinson motor neurological disease thus allowing testing the specificity of PD. To our knowledge, no study has compared the score of the anhedonia subscale of the BDI-II between PD and control group.

Six studies have compared the level of state anhedonia using the SHAPS in PD subjects and controls.

Four studies found significant differences. Lemke et al. [6] reported significantly higher scores on the SHAPS in a group of 626 PD subjects compared with the scores found in 50 healthy volunteers. The sex-ratio and age were not compared between the groups although there were more females and the subjects were older in the PD group. Santangelo et al. [7] reported significantly higher scores on the SHAPS in 74 depressed PD subjects or 76 non-depressed subjects compared with the scores of 74 healthy controls. In this study the three groups were not significantly different for age and sex-ratio but the educational level was unknown. Fujiwara et al. [8] have compared 100 outpatients with PD and 111 healthy subjects recruited in a group of caregivers and care managers. The two groups did not differ significantly for age but there were significantly more females in controls than in PD. The difference regarding gender was not controlled for and the educational level was not rated. The authors reported significantly higher scores on the SHAPS for the PD patients compared with the scores of controls. Jordan et al. [9] reported significantly higher scores on the SHAPS for 50 patients with PD compared with the scores on 42 healthy controls. The controls were significantly older than the PD patients and the status of patients (in or outpatients) or the rate of males were unknown. Thus, the potential effects of age, gender, educational level and status were not controlled for in all of the four studies.

Two studies found no significant difference. Pluck \& Brown [4] have compared 45 PD outpatients with 17 similarly disabled outpatients with osteoarthritis. The two groups were not significantly different for age or sex ratio but the level of education was higher for PD patients than for controls. No significant difference was observed between the two groups on the SHAPS scores. Loas et al. [10] using the same sample as the present study did not find significant differences on the SHAPS between PD patients and non-Parkinson motor neurological disease patients. In this study, gender, age, educational level and status of patients (all the patients were outpatients) were controlled for.

\section{Conclusion}

The results of this study suggest that Parkinson's disease patients were not characterized by high level of anhedonia state, as assessed by the anhedonia subscale of the BDI-II.

\section{References}

[1] Dyduch, A. and Zaluska, M. (2015) Depression in Parkinson's Disease: The Effectiveness and Risk of Pharmacotherapy. Clinical Review. Psychogeriatrics, 15, 147-153. http://dx.doi.org/10.1111/psyg.12078

[2] Loas, G., Krystkowiak, P. and Godefroy, O. (2012) Anhedonia in Parkinson's Disease: An Overview. The Journal of Neuropsychiatry \& Clinical Neurosciences, 24, 444-451. http://dx.doi.org/10.1176/appi.neuropsych.11110332

[3] Assogna, F., Cravello, L., Caltagirone, C. and Spalletta, G. (2011) Anhedonia in Parkinson’s Disease: A Systematic Review of the Literature. Movement Disorders, 26,1825-1834. http://dx.doi.org/10.1002/mds.23815

[4] Pluck, G.C. and Brown, R.G. (2002) Apathy in Parkinson's Disease. Journal of Neurology, Neurosurgery \& Psychiatry, 73, 636-642. http://dx.doi.org/10.1136/jnnp.73.6.636

[5] Isella, V., Iurlaro, S., Piolti, R., Ferrarese, C., Frattola, L., Appollonio, I., et al. (2003) Physical Anhedonia in Parkinson's Disease. Journal of Neurology, Neurosurgery \& Psychiatry, 74, 1308-1311. http://dx.doi.org/10.1136/jnnp.74.9.1308

[6] Lemke, M.R., Brecht, H.M., Koester, J., Kraus, P.H. and Reichmann, H. (2005) Anhedonia, Depression, and Motor Functioning in Parkinson's Disease during Treatment with Pramipexole. The Journal of Neuropsychiatry \& Clinical Neurosciences, 17, 214-220. http://dx.doi.org/10.1176/jnp.17.2.214 
[7] Santangelo, G., Morgante, L., Savica, R., Marconi, R., Grasso, L., Antonini, D., et al. (2009) Anhedonia and Cognitive Impairment in Parkinson's Disease: Italian Validation of the Snaith-Hamilton Pleasure Scale and Its Application in the Clinical Routine Practice during the PRIAMO Study. Parkinsonism \& Related Disorders, 15, 576-581. http://dx.doi.org/10.1016/j.parkreldis.2009.02.004

[8] Fujiwara, S., Kimura, F., Hosokawa, T., Ishida, S., Sugino, M. and Hanafusa, T. (2011) Anhedonia in Japanese Patients with Parkinson's Disease. Geriatrics \& Gerontology International, 11, 275-281. http://dx.doi.org/10.1111/j.1447-0594.2010.00678.x

[9] Jordan, L.L., Zahodne, L.B., Okun, M.S. and Bowers, D. (2013) Hedonic and Behavioral Deficits Associated with Apathy in Parkinson's Disease: Potential Treatment Implications. Movement Disorders, 28, 1301-1304. http://dx.doi.org/10.1002/mds.25496

[10] Loas, G., Duru, C., Godefroy, O. and Krystkowiak, P. (2014) Hedonic Deficits in Parkinson’s Disease: Is Consummatory Anhedonia Specific? Frontiers in Neurology, 5, 24.

[11] Gard, D.E., Germans Gard, M., Kring, A.M. and John, O.P. (2006) Anticipatory and Consummatory Components of the Experience of Pleasure: A Scale Development Study. Journal of Research in Personality, 40, 1086-1102. http://dx.doi.org/10.1016/j.jrp.2005.11.001

[12] Loas, G. and Krystkowiak, P. (2010) The Measurement of Anhedonia in Parkinson's Disease: Psychometric Properties of the Snaith-Hamilton Pleasure Scale (SHAPS) and the Relevance to Distinguish Anticipatory and Consummatory Anhedonias. Movement Disorders, 25, 523-524. http://dx.doi.org/10.1002/mds.22972

[13] Zahodne, L.B., Marsiske, M., Okun, M.S. and Bowers, D. (2012) Components of Depression in Parkinson Disease. Journal of Geriatric Psychiatry and Neurology, 25, 131-137. http://dx.doi.org/10.1177/0891988712455236

[14] Chapman, L.J., Chapman, J.P. and Raulin, M. (1976) Scales for Physical and Social Anhedonia. Journal of Abnormal Psychology, 85, 374-382. http://dx.doi.org/10.1037/0021-843X.85.4.374

[15] Snaith, R.P., Hamilton, M., Morley, S. and Humayan, A. (1995) A Scale for the Assessment of the Hedonic Tone: The Snaith-Hamilton Pleasure Scale. The British Journal of Psychiatry, 167, 99-103. http://dx.doi.org/10.1192/bjp.167.1.99

[16] Joiner, T.E., Brown, J.S. and Metalsky, G.I. (2003) A Test of the Tripartite Model’s Prediction of Anhedonia’s Specificity to Depression: Patients with Major Depression versus Patients with Schizophrenia. Psychiatry Research, 119, 243-250. http://dx.doi.org/10.1016/S0165-1781(03)00131-8

[17] Gard, D.E., Kring, A.M., Germans Gard, M., Horan, W.P. and Green, M.F. (2007) Anhedonia in Schizophrenia: Distinction between Anticipatory and Consummatory Pleasure. Schizophrenia Research, 93, 253-260. http://dx.doi.org/10.1016/j.schres.2007.03.008

[18] Loas, G., Monestes, J.L., Ameller, A., Bubrovszky, M., Yon, V., Wallier, J., Berthoz, S., Corcos, M., Thomas, P. and Gard, D.E. (2009) Traduction et étude de validation de la version française de l'échelle d'expérience temporelle du plaisir (EETP, Temporal Experience of Pleasure Scale [TEPS], Gard et al., 2006): étude chez 125 étudiants et chez 162 sujets présentant un trouble psychiatrique Annales Médico-Psychologiques, Revue Psychiatrique, 167, 641-648. http://dx.doi.org/10.1016/j.amp.2009.09.002

[19] Winer, E.S., Veilleux, J.C. and Ginger, E.J. (2014) Development and validation of the Specific Loss of Interest and Pleasure Scale (SLIPS). Journal of Affective Disorders, 152-154, 193-201. http://dx.doi.org/10.1016/j.jad.2013.09.010

[20] Beck, A.T., Steer, R.A., Ball, R. and Ranieri, W. (1996) Comparison of Beck Depression Inventories-IA and -II in Psychiatric Outpatients. Journal of Personality Assessment, 67, 588-597. http://dx.doi.org/10.1207/s15327752jpa6703_13

[21] Centre de Psychologie Appliquée (1996) Validation de la version française du BDI-II. Editions du Centre de Psychologie Appliquée, Paris. 